\title{
THE BLOW-UP BOUNDARY FOR NONLINEAR WAVE EQUATIONS ${ }^{1}$
}

\author{
LUIS A. CAFFARELLI AND AVNER FRIEDMAN
}

\begin{abstract}
Consider the Cauchy problem for a nonlinear wave equation $\square u=F(u)$ in $N$ space dimensions, $N \leqslant 3$, with $F$ superlinear and nonnegative. It is well known that, in general, the solution blows up in finite time. In this paper it is shown, under some assumptions on the Cauchy data, that the blow-up set is a space-like surface $t=\phi(x)$ with $\phi(x)$ continuously differentiable.
\end{abstract}

Introduction. Consider the nonlinear wave equation

$$
\square u \equiv u_{t t}-\Delta u=F(u)
$$

for $x \in \mathbf{R}^{N}, t>0$, with the initial data

$$
u(x, 0)=f(x), \quad u_{t}(x, 0)=g(x)
$$

for $x \in \mathbf{R}^{N}$. It is well known that if $F(u)$ is nonnegative and superlinear, then, in general, a solution cannot exist for all times. Furthermore, if $T$ is the supremum of all times $s$ such that a classical solution exists for all $0<t \leqslant s$, then

$$
\sup _{x \in \mathbf{R}^{N}}|u(x, t)| \rightarrow \infty \quad \text { if } t \rightarrow T \text {. }
$$

For details see [1-5].

In this paper we are interested in studying the blow-up set, i.e., the set

$$
\Gamma=\partial\{u<\infty\} \cap\{t>0\} .
$$

We assume that $N \leqslant 3$ in order to ensure that the fundamental solution of the d'Alembertian $\square$ is positive (the same assumption is made in $[2,3]$ ). Our main result is that

$\Gamma$ is a $C^{1}$ space-like surface,

that is, $\Gamma$ is given by
$\Gamma: t=\phi(x), \quad$ with $\phi \in C^{1}$ and $|\nabla \phi|<1$.

The conditions on $f, g$ and $F$ are such that they ensure that

$$
u \geqslant 0, \quad \partial u / \partial t>\left|\nabla_{x} u\right|
$$

further, $F(u)$ is convex and $F(u) \sim A u^{p}$ as $u \rightarrow \infty(A>0, p>1)$.

Received by the editors April 2, 1985 and, in revised form, September 17, 1985.

1980 Mathematics Subject Classification. Primary 35L70; Secondary 35L05, 35L67.

${ }^{1}$ This work is partially supported by National Science Foundation Grants 7406375 and MCS 7915171. 
We shall actually establish $\left(0.3^{\prime}\right)$ only in a bounded set $\{|x|<R\}$ ( $R$ is arbitrary), making the corresponding assumptions on $f$ and $g$ in a suitably larger set $\{|x|<R$ $+T\}$.

In $\$ 1$ we give some preliminaries and also state our main theorem. In $\S 2$ we construct a classical solution of $(0.1),(0.2)$ with maximal domain of definition $\Omega$; $\Omega \equiv\{u<\infty\}$. In $\S 3$ we establish a crucial a priori estimate

$$
c \leqslant u_{t t} / u^{2} \leqslant C \quad(0<c<C<\infty)
$$

We also prove that

$$
\begin{aligned}
& u(x, t) \uparrow \infty \text { if } t \uparrow \phi(x), \text { and } \\
& \phi(x) \text { is Lipschitz continuous with coefficient }<1 .
\end{aligned}
$$

In $\S 4$ we proceed to estimate all the first three derivatives of $u$ in terms of powers of $d(x, t)$, the distance to the blow-up boundary. These estimates make it possible to work with blow-up limits with respect to any point $\left(x_{0}, t_{0}\right) \in \Gamma$, i.e., with limits of sequences

$$
\lambda_{n}^{q} u\left(x_{0}+\lambda_{n} x, t_{0}+\lambda_{n} t\right) \quad(q=2 /(p-1))
$$

as $\lambda_{n} \rightarrow 0$. Every limit $v$ is shown to be a convex function.

In $\$ \S 5$ and 6 we show that the blow-up set of any blow-up limit $v$ is planar; in $\$ 5$ we prove this for $N=1$ and in $\S 6$ for $N=2,3$. Using this result we establish, in $\S 7$, the continuous differentiability of the function $\phi(x)$.

1. Preliminaries. Consider the Cauchy problem for the inhomogeneous wave equation

$$
\begin{aligned}
\square w & =h(x, t) & & \left(x \in \mathbf{R}^{N}, t>0\right), \\
w(x, 0) & =f_{0}(x) & & \left(x \in \mathbf{R}^{N}\right), \\
w_{t}(x, 0) & =g_{0}(x) & & \left(x \in \mathbf{R}^{N}\right) .
\end{aligned}
$$

The solution can be represented in the following form: For $N=1$,

$$
\begin{aligned}
w(x, t)= & \frac{1}{2}\left(f_{0}(x+t)+f_{0}(x-t)\right)+\frac{1}{2} \int_{x-t}^{x+t} g_{0}(\xi) d \xi \\
& +\frac{1}{2} \int_{0}^{t} d s \int_{-1}^{1} h(x+(t-s) \eta, s) d \eta
\end{aligned}
$$

for $N=2$,

$$
\begin{aligned}
w(x, t)= & \frac{1}{2 \pi} \int_{|\xi|<t} \frac{g_{0}(x+\xi)}{\sqrt{t^{2}-|\xi|^{2}}} d \xi+\frac{\partial}{\partial t} \frac{1}{2 \pi} \int_{|\xi|<t} \frac{f_{0}(x+\xi)}{\sqrt{t^{2}-|\xi|^{2}}} d \xi \\
& +\frac{1}{2 \pi} \int_{0}^{t}(t-s) d s \int_{|\eta|<t-s} \frac{h(x+\eta, s)}{\sqrt{(t-s)^{2}-|\eta|^{2}}} d \eta
\end{aligned}
$$

for $N=3$,

$$
\begin{aligned}
\left(1.2_{3}\right) u(x, t)= & \frac{t}{4 \pi} \int_{|\xi|=1} g_{0}(x+t \xi) d \omega_{\xi}+\frac{\partial}{\partial t} \frac{t}{4 \pi} \int_{|\xi|=1} f_{0}(x+t \xi) d \omega_{\xi} \\
& +\frac{1}{4 \pi} \int_{0}^{t}(t-s) d s \int_{|\eta|=1} h(x+(t-s) \eta, s) d \omega_{\eta} .
\end{aligned}
$$


These formulas show the positivity of the fundamental solution in dimensions $N \leqslant 3$.

Consider now the nonlinear wave equation

$$
\square u=F(u) \quad\left(x \in \mathbf{R}^{N}, t>0\right),
$$

where $N \leqslant 3$, with the data

$$
\begin{array}{cc}
u(x, 0)=f(x) & \left(x \in \mathbf{R}^{N}\right), \\
u_{t}(x, 0)=g(x) & \left(x \in \mathbf{R}^{N}\right) .
\end{array}
$$

We assume throughout this paper that

$$
\begin{aligned}
& F(u)>0 \quad \text { if } u>0, \\
& F(u) \text { is in } C^{4} \quad \text { for } u>0, \\
& F^{\prime}(u) \geqslant 0, \quad F^{\prime \prime}(u) \geqslant 0 \quad \text { if } u>0, \\
& F(u) u^{-p} \rightarrow A \text { as } u \rightarrow \infty ; A>0,1<p<\infty, \\
& \underset{u \rightarrow \infty}{\limsup } F^{\prime}(u) u^{1-p}<A(p+(p-1) / 2), \\
& \left|F^{(j)}(u)\right| \leqslant C u^{p-j} \quad \text { if } 1 \leqslant u<\infty, 2 \leqslant j \leqslant 4, C>0 .
\end{aligned}
$$

Let $R$ and $T$ be any two positive constants. We shall actually study the behavior of solutions of (1.3)-(1.5) only for $|x|<R, t<T$. Hence, whatever assumptions we impose on the data $f, g$, we need to impose them only for $|x|<R+T$.

Set $B_{\rho}=\{x ;|x|<\rho\}$. The first assumption is

$$
f \text { and } g \text { belong to } C^{4}\left(B_{R+T}\right) \text {. }
$$

The next assumption will be needed to establish the positivity of $u_{1}$ (defined in (2.1)) and subsequently of $u$ :

$\left(1.8_{1}\right) f(x+t)+f(x-t)+\int_{x-t}^{x+t} g(\xi) d \xi \geqslant 0 \quad$ if $|x| \leqslant R, 0 \leqslant t \leqslant T(N=1)$,

$f(x+t \xi)+t g(x+t \xi)>t|\nabla f(x+t \xi)|$ if $|x| \leqslant R, 0 \leqslant t \leqslant T,|\xi| \leqslant 1(N=3)$.

We next impose a condition on $f, g$ in order to ensure that $u_{1, t}$ (and $u_{t}$ ) are positive and, in fact, larger than $\left|\nabla_{x} u_{1}\right|$ (and $\left|\nabla_{x} u\right|$ ):

$$
\begin{array}{rr}
\left(1.9_{1}\right) \quad g(x)>\left(1+\varepsilon_{0}\right)\left|f^{\prime}(x)\right| & \text { if }|x| \leqslant R+T\left(\varepsilon_{0}>0, N=1\right), \\
\left(1.9_{3}\right) \quad g(X)-\left(1+\varepsilon_{0}\right)|\nabla f(X)|+t\left(\Delta f(X)+F(f(X))-\left(1+\varepsilon_{0}\right)|\nabla g(X)|\right) & >t|\nabla g(X)|+\left(1+\varepsilon_{0}\right) t\left|\nabla^{2} f(X)\right| \text { if } X=x+t \xi, \\
& |x| \leqslant R, 0 \leqslant t \leqslant T,|\xi| \leqslant 1\left(\varepsilon_{0}>0, N=3\right) .
\end{array}
$$

We shall not impose direct conditions of the form (1.8), (1.9) in case of $N=2$; it is simpler to apply the method of descent here. Thus, if $N=2$ we define

$$
f\left(x_{1}, x_{2}, x_{3}\right)=f\left(x_{1}, x_{2}\right), \quad g\left(x_{1}, x_{2}, x_{3}\right)=g\left(x_{1}, x_{2}\right),
$$

and write

$$
\begin{array}{ll}
\left(1.8_{3}\right) \text { holds with } f, g \text { defined by }(1.10) & (N=2), \\
\left(1.9_{3}\right) \text { holds with } f, g \text { defined by }(1.10) & (N=2) .
\end{array}
$$


We finally need to impose a condition that implies a blow-up at some point $x$ in $B_{R}$ in time smaller than $T$. For simplicity we state below a condition which ensures a blow-up in time $T_{1}\left(T_{1}<T\right)$ for all $x \in B_{R}$.

Let $w$ be the solution of

$$
\begin{array}{cl}
w^{\prime \prime}(t)=F(w) & (t>0), \\
w(0)=\gamma_{1} & \left(\gamma_{1}>0\right), \\
w^{\prime}(0)=\gamma_{2} & \left(\gamma_{2}>0\right),
\end{array}
$$

where $\gamma_{1}, \gamma_{2}$ are constants such that

$$
w(t) \uparrow \infty \quad \text { if } t \uparrow T_{1} \text { for some } T_{1}<T, \gamma_{1}+T_{1} \gamma_{2}>T_{1} \gamma_{1} .
$$

We shall assume that

$$
f \geqslant 2 \gamma_{1}, \quad \gamma_{1}>|\nabla f|, \quad g \geqslant \gamma_{2} \quad \text { in } B_{R+T} .
$$

Finally, we also assume that

$$
\begin{gathered}
\text { condition }(1.8) \text { holds for } f^{m}, g^{m} \text { with } \\
m=(3 p-1) / 2, \quad m=2 p-1 .
\end{gathered}
$$

For any $\left(x_{0}, t_{0}\right)$ set

$$
\begin{gathered}
K_{-}\left(x_{0}, t_{0}\right)=\left\{(x, t) ;\left|x-x_{0}\right| \leqslant t_{0}-t, t>0\right\}, \\
K_{R, T}=\bigcup_{x \in B_{R}} K_{-}(x, T) .
\end{gathered}
$$

We now state the main result of this paper.

THEOREM 1.1. Let the conditions (1.6)-(1.9) and (1.13), (1.14) hold. Then there exists a classical solution $u(x, t)$ of (1.3)-(1.5) in $K_{R, T} \cap \Omega$, where $\Omega=\{(x, t)$; $\left.x \in B_{R}, 0<t<\phi(x)\right\}$, satisfying

(i) $0<\phi(x)<T$,

(ii) $u(x, t) \uparrow \infty$ if $t \uparrow \phi(x)$,

(iii) $\phi(x)$ is continuously differentiable in $B_{R}$ with $|\nabla \phi(x)| \leqslant 1 /\left(1+\varepsilon_{0}\right)$. The solution is unique in $K_{R, T}$.

The solution $u$ will belong to $C^{3,1}$.

The proof of Theorem 1.1 is given in $\S \S 2-7$.

In $\$ 2$ we construct the solution $u$ in $K_{R, T}$ and in $\S \S 3-7$ we derive various estimates on $u$ near the blow-up boundary $t=\phi(x)$ and establish the $C^{1}$ nature of $\phi(x)$.

2. Construction of the solution. Let $u_{0} \equiv 0$ and define successively a sequence $u_{m}$ by

$$
\begin{gathered}
\square u_{n+1}=F\left(u_{n}\right) \quad\left(x \in \mathbf{R}^{N}, t>0\right), \\
u_{n+1}(x, 0)=f(x) \quad\left(x \in \mathbf{R}^{N}\right), \\
\frac{\partial}{\partial t} u_{n+1}(x, 0)=g(x) \quad\left(x \in \mathbf{R}^{N}\right) .
\end{gathered}
$$


From the representation formulas in (1.2) and from (1.7) it follows that the $u_{m}$ are $C^{4}$ functions in $K_{R, T}$ (defined in (1.14)).

LEMMA 2.1. If (1.6)-(1.8) hold, then

$$
0 \leqslant u_{n}(x, t) \leqslant u_{n+1}(x, t) \text { in } K_{R, T} .
$$

Proof. The inequality $u_{1} \geqslant 0$ follows from the representations (1.2) and (1.8), noting that $F\left(u_{0}\right)=F(0) \geqslant 0$. Proceeding by induction we suppose that $u_{n-1} \leqslant u_{n}$. Then $F\left(u_{n-1}\right) \leqslant F\left(u_{n}\right)$. Representing both $u_{n}$ and $u_{n+1}$ by (1.2), we see that $u_{n} \leqslant u_{n+1}$.

LEMMA 2.2. If (1.6)-(1.9) hold, then

$$
\partial u_{n} / \partial t \geqslant\left(1+\varepsilon_{0}\right)\left|\nabla_{x} u_{n}\right| \text { in } K_{R, T}
$$

PROOF. Set $\lambda=1+\varepsilon_{0}$. For any unit vector $e$ in $\mathbf{R}^{N}$, set $J_{n}=\partial u_{n} / \partial t+\lambda e \cdot \nabla_{x} u_{n}$. Then $\square J_{n+1}=F^{\prime}\left(u_{n}\right) J_{n}$ and

$$
\begin{aligned}
J_{n+1}(x, 0) & =g+\lambda e \cdot \nabla f \equiv \tilde{f}, \\
\frac{\partial}{\partial t} J_{n+1}(x, 0) & =\frac{\partial^{2}}{\partial t^{2}} u_{n+1}(x, 0)+\lambda e \cdot \nabla_{x} \frac{\partial}{\partial t} u_{n+1}(x, 0) \\
& =\Delta f+F(f)+\lambda e \cdot \nabla g \equiv \tilde{g},
\end{aligned}
$$

Notice that $J_{0}=0$, so that $F^{\prime}\left(u_{0}\right) J_{0}=0$. We can now proceed inductively: if $J_{n} \geqslant 0$, then $F^{\prime}\left(u_{n}\right) J_{n} \geqslant 0$ and, using the representation (1.2) for $J_{n+1}$, we find that $J_{n+1} \geqslant 0$; here the condition (1.8) on $\tilde{f}, \tilde{g}$ follows from the condition (1.9) on $f, g$.

Let

$$
u(x, t)=\lim _{n \rightarrow \infty} u_{n}(x, t) \text { in } K_{R, T} .
$$

Since $u(x, t)$ is monotone increasing in $t$, there is a function $\phi(x)$ defined in $B_{R}$ such that $0<\phi(x) \leqslant T$ and, for $x \in B_{R}, 0<t \leqslant T$,

$$
u(x, t)<\infty \text { if } t<\phi(x), \quad u(x, t)=\infty \text { if } t>\phi(x) .
$$

Introduce the set

$$
\Omega=\left\{(x, t) ; x \in B_{R}, 0 \leqslant t<\phi(x)\right\} .
$$

Lemma 2.3. Let (1.6)-(1.9) hold. Then $u$ is a $C^{3,1}$ solution of (1.3)-(1.5) in $\Omega$.

Proof. Fix any positive $R^{\prime}, T^{\prime}$ such that $\overline{K_{R^{\prime}, T^{\prime}}} \subset \Omega$. It suffices to show that $u \in C^{3,1}\left(K_{R^{\prime}, T^{\prime}}\right)$. Notice that

$$
0 \leqslant u_{n} \leqslant C \quad \text { in } K_{R^{\prime}, T^{\prime}}
$$

Let $w_{n}=\partial u_{n} / \partial t$. Then

$$
\square w_{n}=F^{\prime}\left(u_{n-1}\right) w_{n-1}, \quad w_{n}(x, 0)=g(x), \quad \frac{\partial}{\partial t} w_{n}(x, 0)=\Delta f+F(f) .
$$

We shall compare $w_{n}$ in $K_{R^{\prime}, T^{\prime}}$ with the function $W=M e^{B t}$ which satisfies

$$
\square W=B^{2} W, \quad W(x, 0)=M, \quad W_{t}(x, 0)=M B .
$$


Clearly $W>w_{n}$ for small $t$, say for $0 \leqslant t<\eta$, if $M$ is large enough (say $M>\sup _{B_{R}} f$ ).

We claim that $W>w_{n}$ for $K_{R^{\prime}, T^{\prime}}$ provided $M$ and $B$ are large enough, independently of $n$. Indeed otherwise there is a smallest value $t=t_{0}$ such that $W=w_{n}$ at $\left(x_{0}, t_{0}\right)$ for some $x_{0} \in \bar{B}_{R}$. Representing both $W$ and $w_{n}$ at $\left(x_{0}, t_{0}\right)$ by (1.2) and noting that, by $(2.6), B^{2} W>F^{\prime}\left(u_{n-1}\right) w_{n}$ in $K_{-}\left(x_{0}, t_{0}\right)$ if $B$ is large enough, and $M+t M B>f+t g-t|\nabla f|$ (which is the condition $\left(1.8_{3}\right)$ for $W-w_{n}$ ), we deduce that $\left(W-w_{n}\right)\left(x_{0}, t_{0}\right)>0$, a contradiction.

Similarly we can prove that $-w_{n}<W$ in $K_{R^{\prime}, T^{\prime}}$, so that

$$
\left|\partial u_{n} / \partial t\right| \leqslant C \text {. }
$$

The $x_{i}$ derivatives of $u_{n}$ can now be estimated in a similar way, or also by (2.3), (2.7). Thus

$$
\left|D u_{n}\right| \leqslant C \quad \forall n
$$

for any derivative.

Next we apply the above argument to a second derivative $w_{n}=D^{2} u_{n}$. Observe that

$$
\square w_{n}=F^{\prime}\left(u_{n-1}\right) w_{n}+F^{\prime \prime}\left(u_{n-1}\right) D u_{n} D u_{n}
$$

and the last term on the right-hand side is bounded (by (2.8)). Also, $w_{n}(x, 0)$ and $D_{t} w_{n}(x, 0)$ involve only the second derivatives of $f$ and $g$, so that the same comparison function $W$ can again be used provided $M$ and $B$ are large enough. This yields the estimate $\left|D^{2} u_{n}\right| \leqslant C$ in $K_{R^{\prime}, T^{\prime}}$. Similarly we can estimate the third and fourth derivatives of $u_{n}$. Going to the limit we conclude that $u$ is in $C^{3,1}$.

LEMMA 2.4. If (1.6)-(1.9) and (1.13) hold, then

$$
\phi(x)<T \quad \forall x \in B_{R} .
$$

Proof. We compare $u$ with the solution $w$ of (1.11), (1.12). By assumption, $w<u$ for $x \in B_{R^{\prime}}$ and all $t$ small enough; here $R^{\prime}$ is any positive number $<R$. We claim that $w<u$ if $x \in B_{R^{\prime}}, 0<t<T_{1}$. Indeed, otherwise we use the representation (1.2) for both $w$ and $u$ and argue as in the previous lemma. (The condition (1.13) implies the condition (1.8) for $u-w$.) Having thus established that $u>w$ in $B_{R^{\prime}} x\left(0, T_{1}\right)$, (1.12) implies that $\phi(x) \leqslant T_{1}<T$.

ProOF OF UNIQUENESS. Suppose $\bar{u}(x, t), \bar{\phi}(x)$ form another solution satisfying (i)-(iii). Using the representation (1.2), we find, by a standard contraction argument, that

$$
\sup _{x}|(u-\bar{u})(x, t)|=0 \quad\left((x, t) \in K_{R, T}\right)
$$

if $t$ is small enough. Using the fact that $\phi$ and $\bar{\phi}$ are Lipschitz continuous with coefficients smaller than 1 , we can now proceed step-by-step to deduce that $u=\bar{u}$ in all of $K_{R, T} \cap\{t<\phi(x)\} \cap\{t<\bar{\phi}(x)\}$ and then $\phi(x)=\bar{\phi}(x)$ (by (ii)).

3. A $u_{t t}$ estimate. From this section on, we assume that the conditions (1.6)-(1.9) and (1.13) hold, and we shall study the behavior of $u$ near $\Gamma \equiv\{t=\phi(x)\}$ and the regularity of $\phi(x)$. 
For any $\delta_{0}>0$ denote by $\Omega_{\delta_{0}}$ the set of all points in $\Omega$ (defined in (2.5)) whose distance to $\Gamma$ is $\leqslant \delta_{0}$.

THEOREM 3.1. There exists a $\delta_{0}>0$ such that the following estimates hold in $\Omega_{\delta_{0}}$ :

$$
\begin{gathered}
c u^{p} \leqslant u_{t t} \leqslant C u^{p}, \\
c u^{(p+1) / 2} \leqslant u_{t} \leqslant C u^{(p+1) / 2}, \\
c(\phi(x)-t)^{-q} \leqslant u(x, t) \leqslant C(\phi(x)-t)^{-q} \quad(q=2 /(p-1)), \\
c(\phi(x)-t)^{-q-1} \leqslant u_{t}(x, t) \leqslant C(\phi(x)-t)^{-q-1}, \\
c(\phi(x)-t)^{-q-2} \leqslant u_{t t}(x, t) \leqslant C(\phi(x)-t)^{-q-2},
\end{gathered}
$$$$
c(\phi(x)-t)^{-q-1} \leqslant u_{t}(x, t) \leqslant C(\phi(x)-t)^{-q-1},
$$

where $c, C$ are positive constants; in particular, $u(x, t) \uparrow \infty$ if $t \uparrow \phi(x)$.

Proof. We have, for $n \geqslant 2$,

$$
\begin{aligned}
& \square u_{n+1, t t}=F^{\prime}\left(u_{n}\right) u_{n, t t}+F^{\prime \prime}\left(u_{n}\right) u_{n, t}^{2}, \\
& \square F\left(u_{n}\right)=F^{\prime}\left(u_{n}\right) \square u_{n}+F^{\prime \prime}\left(u_{n}\right)\left(u_{n, t}^{2}-\left|\nabla_{x} u_{n}\right|^{2}\right) \\
& =F^{\prime}\left(u_{n}\right) F\left(u_{n-1}\right)+F^{\prime \prime}\left(u_{n}\right)\left(u_{n, t}^{2}-\left|\nabla_{x} u_{n}\right|^{2}\right), \\
& \square u_{n+1, t}=F^{\prime}\left(u_{n}\right) u_{n, t} .
\end{aligned}
$$

Setting $J_{n+1}=u_{n+1, t t}-F\left(u_{n}\right)+M u_{n+1, t}(M>0)$ we get

$$
\square J_{n+1}=F^{\prime}\left(u_{n}\right) J_{n}+F^{\prime \prime}\left(u_{n}\right)\left|\nabla_{x} u_{n}\right|^{2} \text {. }
$$

Also

$$
\begin{aligned}
& J_{n+1}(x, 0)=f_{1}+M g, \\
& J_{n+1, t}(x, 0)=g_{1}+M(\Delta f+F(f)),
\end{aligned}
$$

where $f_{1}, g_{1}$ are functions independent of $n$ and $M$.

Representing $J_{n+1}$ by (1.2) and assuming inductively that $J_{n} \geqslant 0$ (note that $J_{2} \geqslant 0$ if $M$ is sufficiently large) we conclude that $J_{n+1} \geqslant 0$ provided (1.8) holds for the data in (3.7). Now, for $N=1$ the condition (1.8) for (3.7) with $M$ large reduces to

$$
g(x+t)+g(x-t)+\int_{x-t}^{x+t}\left(f^{\prime \prime}(\xi)+F(f(\xi))\right) d \xi>0
$$

which is a consequence of $\left(1.9_{1}\right)$. For $N=3$ the condition (1.8) for $J_{n+1}$ (with $M$ large) reduces to

$$
g(x+t \xi)+t(\Delta f(x+t \xi)+F(f(x+t \xi)))>t|\nabla g(x+t \xi)|
$$

which is a consequence of $\left(1.9_{3}\right)$. We conclude that, indeed,

$$
J_{n, 1} \geqslant 0 \text {. }
$$

Similarly, setting

$$
\tilde{J}_{n+1}=\frac{\varepsilon_{0}}{1+\varepsilon_{0}} u_{n+1, t t}-F\left(u_{n}\right)-M u_{n+1, t}
$$


we get, analogously to (3.6),

$$
\square \tilde{J}_{n+1}=F^{\prime}\left(u_{n}\right) \tilde{J}_{n}-\left(\frac{1}{1+\varepsilon_{0}} u_{n, t}^{2}-\left|\nabla_{x} u_{n}\right|^{2}\right) F^{\prime \prime}\left(u_{n}\right) \leqslant F^{\prime}\left(u_{n}\right) \tilde{J}_{n},
$$

where Lemma 2.2 was used. Further, condition (1.8) holds for the initial data of $-\tilde{J}_{n+1}$. We can therefore deduce inductively that

$$
\tilde{J}_{n+1} \leqslant 0 \text {. }
$$

From (3.9) and some of the assumptions on $F$ in (1.6) we deduce, after recalling that $u_{n} \leqslant u_{n+1}$, that the function $w=u_{n+1}$ satisfies $w_{t t} \leqslant C w^{p}+M w_{t}$. Multiplying by $w_{t} e^{-2 M t}$ and integrating we easily get $w_{t}^{2} \leqslant C w^{p+1}+C_{1}$ or $d w /\left[C w^{p+1}+C_{1}\right]^{1 / 2}$ $\leqslant d t$.

It follows that

$$
\int_{u_{n+1}\left(x_{0}, \tau\right)}^{u_{n+1}\left(x_{0}, t\right)} \frac{d s}{\left[C s^{p+1}+C_{1}\right]^{1 / 2}} \leqslant t-\tau .
$$

Taking $t=t_{0}+\varepsilon$, where $t_{0}=\phi\left(x_{0}\right)$, and letting $n \rightarrow \infty$ we get

$$
\int_{u\left(x_{0}, \tau\right)}^{\infty} \frac{d s}{\left[C s^{p+1}+C_{1}\right]^{1 / 2}} \leqslant t_{0}+\varepsilon-\tau .
$$

Letting $\varepsilon \rightarrow 0$ and evaluating the integral we obtain, for some $c>0$,

$$
u\left(x_{0}, \tau\right) \geqslant \frac{c}{\left(t_{0}-\tau\right)^{q}} \quad\left(q=\frac{2}{p-1}\right)
$$

which shows that $u\left(x_{0}, t\right) \rightarrow \infty$ if $t \rightarrow \phi\left(x_{0}\right)$.

We now take $n \rightarrow \infty$ in (3.9) and get

$$
u_{t t} \leqslant F(u)+M u_{t} .
$$

Proceeding as before (with $w$ ) we find that

$$
u_{t}^{2} \leqslant C u^{p+1}+C_{1} .
$$

Hence (3.12) can be simplified to

$$
u_{t t} \leqslant C u^{p}
$$

with another constant $C$.

Similarly, using (3.8) we derive the estimate

$$
u_{t t} \geqslant c_{0} u^{p}-C_{1} \quad\left(c_{0}>0, C_{1}>0\right),
$$

and therefore, by (3.11),

$$
u_{t t} \geqslant c u^{p} \quad(c>0)
$$

in some $\Omega_{\delta_{0}}$. Multiplying both sides of (3.15) by $u_{t}$ and integrating we find that

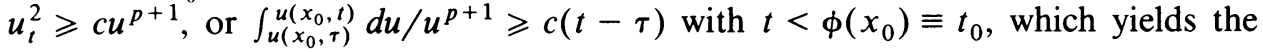
estimate $u\left(x_{0}, \tau\right) \leqslant c /\left(t_{0}-\tau\right)^{q}$ thus complementing (3.11).

The remaining assertions of the theorem follow immediately from the estimates already derived. 
From Lemma 2.2 we have

$$
\left|\nabla_{x} u\right| \leqslant \frac{1}{1+\varepsilon_{0}} u_{t}
$$

this is valid in $K_{R^{\prime}, T} \cap \Omega$ for some $R^{\prime}>R$ (since $\phi(x) \leqslant T_{1}<T$ if $x \in B_{R^{\prime}}$, by the proof of Lemma 2.4). Since $u(x, t)<\infty$ if $t<\phi(x)$ and $u(x, t)=\infty$ if $t=\phi(x)$, we easily obtain

COROllaRY 3.2. $\phi(x)$ is Lipschitz continuous with coefficient $\leqslant 1 /\left(1+\varepsilon_{0}\right)$, i.e.,

$$
\left|\phi(x)-\phi\left(x^{\prime}\right)\right| \leqslant \frac{\left|x-x^{\prime}\right|}{1+\varepsilon_{0}} \quad \text { if } x, x^{\prime} \in B_{R^{\prime}}
$$

Definition 3.1. We denote by $d(x, t)$ the distance from a point $(x, t)$ in $\Omega$ to the blow-up set $\Gamma$.

In view of Corollary 3.2,

$$
(\phi(x)-t) / \sqrt{2} \leqslant d(x, t) \leqslant \phi(x)-t .
$$

From (3.3)-(3.5) and (3.16), (3.18) we get

Corollary 3.3. For some positive constants $c, C$ the following estimates hold in $\Omega_{\delta_{0}}$ :

$$
\begin{gathered}
c \leqslant u d^{q} \leqslant C, \\
c \leqslant u_{t} d^{q+1} \leqslant C, \\
c \leqslant u_{t t} d^{q+2} \leqslant C, \\
\left|\nabla_{x} u\right| \leqslant C d^{-(q+1)} .
\end{gathered}
$$

\section{Blow-up limits.}

LEMMA 4.1. The following estimates hold in $\Omega$ :

$$
\left|D^{\alpha} u\right| \leqslant C u^{p+(|\alpha|-2) / q} \leqslant C d^{-(p q+|\alpha|-2)} \quad(0 \leqslant|\alpha| \leqslant 4),
$$

where $D^{\alpha}$ is any space-time derivative of order $|\alpha|$ and $C$ is a constant.

Proof. Note that $D u$ and $D_{t t} u$ have already been estimated in Theorem 3.1. To estimate any spacial derivative $D_{l l} u$ we proceed as in the proof of (3.9) working with

$$
\tilde{J}_{n+1}=\frac{\varepsilon_{0}}{1+\varepsilon_{0}} u_{n+1, l l}-F\left(u_{n}\right)-M u_{n+1, t} \text {. }
$$

This yields the estimate

$$
\left|D_{l l} u\right| \leqslant C u^{p} \text {. }
$$

Consider next any third order space-time derivative $D^{3}$ and for simplicity take it to be a pure derivative. Then

$$
\square D^{3} u=F^{\prime}(u) D^{3} u+3 F^{\prime \prime}(u) D u D^{2} u+F^{(3)}(u)(D u)^{3} .
$$

On the other hand, if $m=p+1 / q$,

$$
\square u^{m}=m \frac{F(u)}{u} u^{m}+m(m-1) u^{m-2}\left(u_{t}^{2}-\left|\nabla_{x} u\right|^{2}\right) \geqslant m \frac{F(u)}{u} u^{m}
$$

by Lemma 2.2 . 
The last two conditions in (1.6) imply that, for some $\varepsilon>0$,

$$
F^{\prime}(u) \leqslant(m-\varepsilon) \frac{F(u)}{u} \text { if } u \geqslant B_{\varepsilon}
$$

and

$$
\begin{aligned}
\left|F^{\prime \prime}(u) D u D^{2} u\right| & \leqslant C u^{p-2}|D u|\left|D^{2} u\right| \\
& \leqslant C u^{3 p-2-1 / q}=C u^{p-1+m} \leqslant C_{1} m \frac{F(u)}{u} u^{m}, \\
\left|F^{(3)}(u)(D u)^{3}\right| & \leqslant C u^{p-2}|D u|^{3} \leqslant C u^{p-3+3 p-3 / q} \\
& =C u^{p-1+m} \leqslant C_{1} m \frac{F(u)}{u} u^{m},
\end{aligned}
$$

where (4.1) for $|\alpha| \leqslant 2$ was used; here $B_{\varepsilon}, C$ and $C_{1}$ are suitable constants. It follows that if $J=M u^{m}+D^{3} u$, where $M$ is a sufficiently large positive constant, then $\square J \geqslant F^{\prime}(u) J$. In view of (1.14), the initial data for $J$ at $t=0$ satisfy the condition (1.8) if $M$ is large enough. We can now easily deduce that

$$
J>0 \text { in } \Omega \text {. }
$$

Indeed, otherwise there is a smallest value $t=t_{0}$ such that $J\left(x_{0}, t_{0}\right)=0$ for some $x_{0} \in B_{R}$. Representing $J\left(x_{0}, t_{0}\right)$ by $(1.2)$ we find that $J\left(x_{0}, t_{0}\right)>0$; a contradiction.

Having proved (4.3) we conclude that (4.1) holds for $|\alpha|=3$. The proof for $|\alpha|=4$ is formally similar, working with $D^{4} u+M u^{m^{\prime}}$, where $m^{\prime}=p+2 / q$. Actually, since $u$ is only in $C^{3,1}$, we work first with the $D^{\alpha} u_{n+1}+M u_{n}^{m}$ for $|\alpha|=3$, then with $D^{\alpha} u_{n+1}+M u_{n}^{m^{\prime}}$ for $|\alpha|=4$ and finally let $n \rightarrow \infty$.

Let $\left(x_{0}, t_{0}\right)$ be any point of $\Gamma$, and introduce the scaled functions

$$
u_{\lambda}(x, t)=\lambda^{q} u\left(x_{0}+\lambda x, t_{0}+\lambda t\right) \quad(\lambda>0) .
$$

Any sequence $\left\{u_{\lambda_{n}}\right\}$ with $\lambda_{n} \rightarrow 0$ is called a blow-up sequence.

Denote by $\left\{t=\phi_{\lambda}(x)\right\}$ the blow-up set for $u_{\lambda}$, i.e.,

$$
\phi_{\lambda}(x)=\left(\phi\left(x_{0}+\lambda x\right)-\phi\left(x_{0}\right)\right) / \lambda \text {. }
$$

Let $\Omega_{\lambda}=\left\{(x, t) ;\left(x_{0}+\lambda x, t_{0}+\lambda t\right) \in \Omega\right\}$ and denote by $d_{\lambda}(x, t)$ the distance from a point $(x, t)$ in $\Omega_{\lambda}$ to the blow-up set $\Gamma_{\lambda}=\left\{t=\phi_{\lambda}(x)\right\}$.

From Lemma 4.1 we obtain

$$
\left|D^{\alpha} u_{\lambda}\right| \leqslant D u_{\lambda}^{p+(|\alpha|-2) / q} \leqslant C d_{\lambda}^{-(p+q+|\alpha|-2)} \quad(0 \leqslant|\alpha| \leqslant 4),
$$

and from Theorem 3.1 and (3.16), (3.17) we have

$$
\begin{aligned}
& c u_{\lambda}^{p} \leqslant D_{t t} u_{\lambda} \leqslant C u_{\lambda}^{p}, \\
& C u_{\lambda}^{(p+1) / 2} \leqslant D_{t} u_{\lambda} \leqslant C u_{\lambda}^{(p+1) / 2} \\
& \left|\nabla_{x} u_{\lambda}\right| \leqslant \frac{1}{1+\varepsilon_{0}} D_{t} u_{\lambda}, \\
& \left|\phi_{\lambda}(x)-\phi_{\lambda}\left(x^{\prime}\right)\right| \leqslant \frac{1}{1+\varepsilon_{0}}\left|x-x^{\prime}\right| .
\end{aligned}
$$


It follows that any blow-up sequence $u_{\lambda_{n}}$ has a subsequence for which

$$
\phi_{\lambda_{n}}(x) \rightarrow \phi_{0}(x)
$$

uniformly in compact sets,

$$
u_{\lambda_{n}} \rightarrow v \text { in } C^{3, \alpha} \forall 0<\alpha<1
$$

in any compact subset of

$$
\Omega_{0} \equiv\left\{(x, t) ; x \in \mathbf{R}^{N}, t<\phi_{0}(x)\right\},
$$

and

$$
\begin{gathered}
\square v=A v^{p} \quad \text { in } \Omega_{0}, \\
\left|D^{\alpha} v\right| \leqslant C v^{(p+|\alpha|-2) / q} \quad \text { in } \Omega_{0}(|\alpha| \leqslant 4), \\
c v^{p} \leqslant v_{t t} \leqslant C v^{p} \quad \text { in } \Omega_{0}, \\
c v^{(p+1) / 2} \leqslant v_{t} \leqslant C v^{(p+1) / 2} \quad \text { in } \Omega_{0}, \\
c d_{0}^{-q} \leqslant v \leqslant C d_{0}^{-q} \quad \text { in } \Omega_{0}, \\
\left|\nabla_{x} v\right| \leqslant \frac{1}{1+\varepsilon_{0}} v_{t} \quad \text { in } \Omega_{0}, \\
\left|\phi_{0}(x)-\phi_{0}\left(x^{\prime}\right)\right| \leqslant \frac{1}{1+\varepsilon_{0}}\left|x-x^{\prime}\right| \quad\left(x, x^{\prime} \in \mathbf{R}^{N}\right),
\end{gathered}
$$

where $c, C$ are positive constants and $d_{0}(x, t)$ denotes the distance from a point $(x, t)$ of $\Omega_{0}$ to $\partial \Omega_{0}$.

Definition 4.1. The function $v$ is called a blow-up limit of $u$ with respect to the center $\left(x_{0}, t_{0}\right)$.

We conclude this section by proving

Lemma 4.2. Any blow-up limit is convex.

Proof. Let $v$ be a blow-up limit and let $J=v_{l l}+\eta v_{t}(\eta>0)$, where $v_{l l}$ is any pure second derivative of $v$ (in any direction in $R^{N+1}$ ). Then

$$
\square J=p A v^{p-1} J+p(p-1) A v^{p-2} v_{l}^{2} .
$$

Fix a point $(\bar{x}, \bar{t})$ in $\Omega_{0}$ and consider $J$ in $K_{-}(\bar{x}, \bar{t})$. Because of (4.17)

$$
c \leqslant \frac{d_{0}(x, t)}{|t|} \leqslant C \quad \text { if }(x, t) \in K_{-}(\bar{x}, \bar{t}), t \rightarrow-\infty,
$$

where $c, C$ are positive constants.

We claim:

$$
J>0 \text { in } K_{-}(\bar{x}, \bar{t}) .
$$

It will suffice to prove it for $N=3$. By (4.12), (4.14) and (4.19), $J=\eta v_{t}(1+O(1 /|t|))$ in $K_{-}(\bar{x}, \bar{t})$ as $t \rightarrow-\infty$, so that $J>0$ in $K_{-}(\bar{x}, \bar{t})\{t<-\sigma\}$ for some sufficiently large $\sigma$.

If (4.20) is not true, then there must exist a point $\left(x_{0}, t_{0}\right)$ in $K_{-}(\bar{x}, \bar{t})$ with smallest $t_{0}$ such that

$$
J\left(x_{0}, t_{0}\right)=0
$$


Consider the cones $K_{-}\left(x_{0}, t_{0}\right)$ and $K_{+}\left(x_{0}, t_{1}\right)=\left\{(x, t) ;\left|x-x_{0}\right|<t-t_{1}, t\right\rangle$ $\left.t_{1}\right\}$ when $t_{1}=t_{0}-2 M$ and $M$ is a positive constant to be determined. We represent $J\left(x_{0}, t_{0}\right)$ in $K_{-}\left(x_{0}, t_{0}\right) \cap\left\{t>t_{0}-M\right\}$ and $J\left(x_{0}, t_{1}\right)$ in $K_{+}\left(x_{0}, t_{1}\right) \cap\left\{t<t_{0}-\right.$ $M$ \} by (1.2) and, by adding, obtain

$$
\begin{aligned}
J\left(x_{0}, t_{0}\right)+J\left(x_{0}, t_{1}\right) \\
=\frac{1}{2 \pi} \int_{|\xi|=1}\left(r f_{r}\right) d \omega_{\xi}+\frac{1}{2 \pi} \int_{|\xi|=1} J\left(x_{0}+M \xi, t_{0}-M\right) d \omega_{\xi} \\
\quad+\frac{1}{4 \pi} \int_{0}^{M}(M-s) d s \int_{|\eta|=1} J\left(x_{0}+(M-s) \eta, s+t_{0}-M\right) d \omega_{\eta} \\
\quad+\frac{1}{4 \pi} \int_{0}^{M}(M-s) d s \int_{|\eta|=1} J\left(x_{0}+(M-s) \eta,-s+t_{1}+M\right) d \omega_{\eta} \\
\equiv I_{0}+I_{1}+I_{2}+I_{3},
\end{aligned}
$$

where $f$ is equal to $J\left(x_{0}+x, t_{0}-M\right) \equiv J_{0}(x)$ evaluated at $x=M \xi, f_{r}$ is the radial derivative of $J_{0}(x)$ evaluated at $M \xi$, and $r=M$. By (4.12), (4.14), (4.15), (4.19),

$$
J\left(x_{0}, t_{1}\right)=O\left(\frac{1}{M^{(p+1) /(p-1)}}\right) \rightarrow 0 \text { if } M \rightarrow \infty .
$$

On the other hand, $I_{0}=O\left(M^{1-p}\right) \rightarrow 0$ if $M \rightarrow \infty$, by (4.12), (4.15), (4.19). Also $I_{1}>0, I_{2}>0, I_{3}>0$; furthermore, $I_{2} \geqslant c>0$ where $c$ is independent of $M$. It follows from (4.22) with $M$ large enough that $J\left(x_{0}, t_{0}\right)>0$, a contradiction to (4.21). This completes the proof of (4.20) and, in particular, $v_{l l}(\bar{x}, \bar{t})+\eta v_{t}(\bar{x}, \bar{t}) \geqslant 0$. Taking $\eta \rightarrow 0$, we get $v_{l l}(\bar{x}, \bar{t}) \geqslant 0$. Since $(\bar{x}, \bar{t})$ and $l$ are arbitrary, the lemma follows.

Set

$$
\Gamma_{0}=\partial \Omega_{0} \equiv\left\{(x, t) ; t=\phi_{0}(x)\right\} .
$$

In the next two sections it will be proved that $\Gamma_{0}$ is a plane (a line, if $N=1$ ).

5. $\Gamma_{0}$ is linear $(N=1)$. In this section we take $N=1$.

We shall need the special solutions of

$$
\square V=A V^{p}
$$

with blow-up boundary

$$
\{(x, t) ; t=\alpha x,-\infty<x<\infty\}, \quad \alpha \text { real, }
$$

given by

$$
V_{\alpha}(x, t)=C_{\alpha}(\alpha x-t)^{-q}, \quad C_{\alpha}^{p-1}=\frac{1}{A} q(q+1)\left(1-\alpha^{2}\right) .
$$

We refer to them as linear solutions.

REMARK 5.1. If $v$ is a convex solution of (4.11)-(4.16) and $\Omega_{0}$ is half a plane, then $v$ is a linear solution. Indeed, let $l$ be a line in $\Omega_{0}$ parallel to $\partial \Omega_{0}$. Then, along $l, v$ is bounded (by (4.15)) and convex; hence $v$ is constant along $l$. Since $v$ also satisfies (5.1), it easily follows that it has the form (5.3) with some $\alpha,|\alpha|<1$. 
LEMMA 5.1. $\Gamma_{0}$ is a straight line and $v=V_{\alpha}$ for some $\alpha,|\alpha|<1$.

Proof. From Lemma 4.2 we have that, for any positive $\gamma$, the set $\{v \leqslant \gamma\}$ is convex. Thus the level curves $\{v=\gamma\}$ are given by $t=\psi_{\gamma}(x)$ with $\psi_{\gamma}(x)$ concave, and also $\phi_{0}(x)$ is a concave function.

We introduce an implosion (i.e., a blow-up at $\infty)$ by $v_{\lambda}(x, t)=\lambda^{q} v(\lambda x, \lambda t)$ with $\lambda \rightarrow \infty$. As in the case of blow-up limits, for any sequence of $\lambda$ 's there is a subseqeunce $\lambda_{n} \rightarrow \infty$ such that $v_{\lambda_{n}} \rightarrow w$ uniformly in compact subsets of $\hat{\Omega} \equiv\{w$ $<\infty$ ) and (4.11)-(4.16) hold for $w$. Further, since $\phi_{0}(x)$ is concave, the blow-up boundary $\hat{\Gamma}$ of $w$ is given by two rays, $l_{\alpha}$ and $l_{\beta}$, with slopes $\alpha$ and $\beta$, and $\beta \leqslant \alpha$; also $|\alpha|<1,|\beta|<1$. If

$$
\alpha=\beta,
$$

then, by Remark 5.1, $\phi_{0}(x)$ is a linear function. In order to prove (5.4) we may take, for definiteness,

$$
0<\beta \leqslant \alpha<1
$$

so that

$$
l_{\alpha}=\{t=\alpha x, x<0\}, \quad l_{\beta}=\{t=\beta x, x>0\} .
$$

Introduce the sectors

$$
\begin{aligned}
& \Omega_{\alpha}=\text { the sector bounded by the rays } l_{\alpha} \text { and }\left\{t=\frac{x}{\alpha}, x<0\right\}, \\
& \tilde{\Omega}_{\alpha}=\text { the sector bounded by the rays } l_{\alpha} \text { and }\{t=-x, x>0\} .
\end{aligned}
$$

We introduce directional derivatives

$$
D_{\sigma}=\alpha D_{t}+D_{x} \quad(\text { space-like }), \quad D_{\tau}=D_{t}+\alpha D_{x} \quad \text { (time-like) }
$$

we refer to the direction determined by $D_{\tau}$ as the conjugate to $l_{\alpha}$ (or the conjugate normal to $l_{\alpha}$ ).

We shall need several lemmas.

\section{LEMMA 5.2. There holds}

$$
w \leqslant V_{\alpha} \text { in } \Omega_{\alpha}
$$

Proof. Since

$$
D_{\tau}^{2}-D_{\sigma}^{2}=\left(D_{t}+\alpha D_{x}\right)^{2}-\left(\alpha D_{t}+D_{x}\right)^{2}=\left(1-\alpha^{2}\right) \square
$$

we have

$$
\left(D_{\tau}^{2}-D_{\sigma}^{2}\right) w=A\left(1-\alpha^{2}\right) w^{p} .
$$

Also, since $D_{\sigma} V_{\alpha}=\left(\alpha D_{t}+D_{x}\right) V_{\alpha}=0$,

$$
D_{\tau}^{2} V_{\alpha}=A\left(1-\alpha^{2}\right) V_{\alpha}^{p}
$$

Comparing with (5.9) and recalling that, by the convexity of $w, D_{\sigma}^{2} w \geqslant 0$, we see that

$$
D_{\tau}^{2}\left(w-V_{\alpha}\right) \geqslant A\left(1-\alpha^{2}\right)\left(w^{p}-V_{\alpha}^{p}\right)
$$


Let $V_{\alpha}^{\varepsilon}$ be an $\varepsilon$-translation of $V_{\alpha}$ with blow-up boundary $\{t=\alpha x-\varepsilon\}$.

Let $m$ be any ray in the direction $-\tau$ with initial point on $\{t=\alpha x-\varepsilon\}$. Then $w-V_{\alpha}^{\varepsilon}=-\infty$ at the initial point of $m$, and $w-V_{\alpha}^{\varepsilon} \rightarrow 0$ as $(x, t)$ goes to infinity along $m$. Since (5.10) holds also with $V_{\alpha}$ replaced by $V_{\alpha}^{\varepsilon}$, we can apply the maximum principle to conclude that $w-V_{\alpha}^{\varepsilon} \leqslant 0$ along $m$. Taking $\varepsilon \rightarrow 0$, assertion (5.8) follows.

LEMMA 5.3. There holds

$$
w(x-\lambda, t-\alpha \lambda) \rightarrow V_{\alpha}(x, t) \quad \text { as } \lambda \rightarrow \infty
$$

uniformly in any compact subset of $\tilde{\Omega}=\{t<\alpha x,-\infty<x<\infty\}$.

Proof. Set $\tilde{w}_{\lambda}(x, t)=w(x-\lambda, t-\alpha \lambda)$. By the $C^{3,1}$ estimates on $w$ it follows that for any sequence of $\lambda$ 's converging to $\infty$ there is a subsequence $\lambda_{n}$ such that $\tilde{w}_{\lambda_{n}}(x, t) \rightarrow W$ uniformly in compact subsets of $\tilde{\Omega}$, and

$$
\square W=A W^{p} \quad \text { in } \tilde{\Omega} \text {; }
$$

furthermore, $W$ is convex and satisfies (4.12)-(4.16), and its blow-up boundary is $\partial \tilde{\Omega}$.

By Remark 5.1 we now deduce (recalling the definition of $\tilde{\Omega}$ ) that $W=V_{\alpha}$, and the assertion (5.11) follows.

\section{LEMMA 5.4. There holds}

$$
w=V_{\alpha} \text { in } \Omega_{\alpha} \text {. }
$$

Proof. Consider any level curve $\Lambda_{\gamma}:\{w=\gamma\}$. By Lemma $5.3 w-V_{\alpha} \rightarrow 0$ along $\Lambda_{\gamma}$ as $x \rightarrow-\infty$. Hence

$$
\gamma=\hat{C}_{\alpha} \theta^{-q}, \quad \hat{C}_{\alpha}=C_{\alpha}\left(1+\alpha^{2}\right)^{-q / 2},
$$

where

$$
\theta=\lim \operatorname{dist}\left((x, t), l_{\alpha}\right) \quad \text { as }(x, t) \in \Lambda_{\gamma}, \quad x \rightarrow-\infty .
$$

We claim that

$$
\Lambda_{\gamma} \text { is parallel to } l_{\alpha} \text { in } \Omega_{\alpha} \text {. }
$$

Indeed, since $\Lambda_{\gamma}$ is convex to $l_{\alpha}$, if the assertion (5.15) is not true then there exist points $(\tilde{x}, \tilde{t})$ in $\Lambda_{\gamma} \cap \Omega_{\alpha}$ whose distance $\tilde{\theta}$ to $l_{\alpha}$ is larger than $\theta$. It follows that

$$
V_{\alpha}(\tilde{x}, \tilde{t})=\hat{C}_{\alpha} \tilde{\theta}^{-q}<\hat{C}_{\alpha} \theta^{-q}=\gamma=w(\tilde{x}, \tilde{t}),
$$

a contradiction to Lemma 5.2 .

From (5.15) we quickly deduce that $w=V_{\alpha}$.

We next extend Lemma 5.4 by proving:

\section{LEMMA 5.5. There holds}

$$
w=V_{\alpha} \text { in } \tilde{\Omega}_{\alpha}
$$

Proof. Since $V_{\alpha}$ is constant in every direction parallel to $l_{\alpha}$ whereas $w$ is convex, we immediately deduce from Lemma 5.4 that

$$
w \geqslant V_{\alpha} \text { in } \tilde{\Omega}_{\alpha} .
$$


Take any square $T$ in $\tilde{\Omega}_{\alpha}$ with one of the diagonals parallel to the $t$-axis, and denote its vertices by $P_{i}$, so that $P_{1}=\left(x_{0}, t_{1}\right), P_{2}=\left(x_{0}, t_{2}\right)$ with $t_{2}<t_{1}$. By $\left(1.2_{1}\right)$

$$
\begin{gathered}
w\left(P_{1}\right)+w\left(P_{2}\right)=w\left(P_{3}\right)+w\left(P_{4}\right)+\frac{1}{2} \int_{T} A w^{p}, \\
V_{\alpha}\left(P_{1}\right)+V_{\alpha}\left(P_{2}\right)=V_{\alpha}\left(P_{3}\right)+V_{\alpha}\left(P_{4}\right)+\frac{1}{2} \int_{T} A V_{\alpha}^{p} .
\end{gathered}
$$

If $T$ is chosen so that $P_{1}$ and $P_{2}$ belong to $\Omega_{\alpha}$, then the left-hand sides of (5.18) and (5.19) coincide. Since also $w \geqslant V_{\alpha}$ in $T$, it follows, by comparing the right-hand sides of (5.18), (5.19), that $w=V_{\alpha}$ in $T$.

By varying $T$ we can cover some $\varepsilon$-neighborhood of the ray $\{t=x / \alpha, x<0\}$. Proceeding step-by-step we can cover in this fashion all of $\tilde{\Omega}_{\alpha}$, and (5.16) follows.

COMPLETION OF THE PROOF OF LEMMA 5.1. If (5.4) is not true, then $\beta<\alpha$ and $G \equiv \tilde{\Omega}_{\alpha} \cap \tilde{\Omega}_{\beta} \neq \varnothing$, where $\tilde{\Omega}_{\beta}$ is the sector bounded by the ray $l_{\beta}$ and $\{t=x$, $x<0\}$. Similarly to Lemma 5.5 we have $w=V_{\beta}$ in $\tilde{\Omega}_{\beta}$, so that $V_{\alpha}=V_{\beta}$ in $G$, a contradiction.

Having proved that $\alpha=\beta$, it follows that $\Gamma_{0}$ is linear and $w=V_{\alpha}$.

6. $\Gamma_{0}$ linear $(N \leqslant 3)$. In this section we extend Lemma 5.1 to $N=2,3$. It will be convenient to state the result in a slightly more general form:

LEMMA 6.1. Let $v$ be any solution of (4.10)-(4.17) which is convex. Then $\partial \Omega_{0}$ is a hyperplane and, for a suitable rotation of the $x_{i}$ coordinates,

$$
v(x, t)=V_{\alpha}\left(x_{N}, t\right) \quad \text { for some } \alpha \in[0,1) .
$$

Proof. For $N=1$ the proof is the same as the proof of Lemma 5.1. We may therefore proceed by induction on $N$.

Introduce implosions $v_{\lambda}$ as in the case $N=1$ and take any $\operatorname{limit} w=\lim _{\lambda_{n} \rightarrow \infty} v_{\lambda_{n}}$. Since $v$ is convex, the same is true of $w$; further, the blow-up set of $w$ is a convex cone $\hat{\Gamma}$ with vertex at the origin. To prove that $\partial \Omega_{0}$ is a hyperplane it suffices to show that

$$
\text { the convex cone } \hat{\Gamma} \text { is a hyperplane. }
$$

Let $l$ be any generatrix of $\hat{\Gamma}$, and $l_{0}$ the straight line containing $l$. For simplicity we take

$$
l=\left\{x_{i}=0 \text { if } 1 \leqslant i \leqslant N-1, t=\alpha x_{N}, x_{N}<0\right\},
$$

where $\alpha>0$; clearly $\alpha<1$. Set

$$
\begin{aligned}
& \hat{\Omega}=\{w<\infty\}, \\
& x^{\lambda}=\left(x_{1}, x_{2}-\lambda\right) \quad \text { if } N=2, \quad x^{\lambda}=\left(x_{1}, x_{2}, x_{3}-\lambda\right) \quad \text { if } N=3, \\
& \hat{\Omega}^{\lambda}=\left\{(x, t) ;\left(x^{\lambda}, t-\alpha \lambda\right) \in \hat{\Omega}\right\} \\
& \tilde{w}^{\lambda}(x, t)=w\left(x^{\lambda}, t-\alpha \lambda\right) .
\end{aligned}
$$

Then, for any sequence of $\lambda$ 's increasing to $\infty$ there is a subsequence $\lambda_{n}$ such that $\lim _{\lambda_{n} \rightarrow \infty} \hat{\Omega}^{\lambda_{n}} \tilde{\Omega}$ and $\tilde{w}^{\lambda_{n}} \rightarrow W$ uniformly in compact subsets of $w$; further,

$\tilde{\Omega}$ is a cylinder $l_{0} \times \Omega_{0}$ where $\Omega_{0}$ is a convex set. 
To prove (6.4) denote by $e$ a unit vector in the $l_{0}$ direction. If $X \in \tilde{\Omega}$, then clearly $X+\lambda e \in \tilde{\Omega}$ for any real $\lambda$, and thus $\tilde{\Omega}$ is a cylinder $l_{0} \times \Omega_{0}$. Since the sets $\hat{\Omega}^{\lambda_{n}}$ are increasing and convex, their limit $\tilde{\Omega}$ is also convex; hence $\Omega_{0}$ is convex.

Observe that $W$ is a convex function satisfying (5.12) and (4.12)-(4.16).

Set $\tilde{\Gamma}=\partial \tilde{\Omega}, \tilde{\Gamma}_{0}=\partial \Omega_{0}$.

Introduce time-like and space-like directional derivatives $D_{\tau}=D_{t}+\alpha D_{x_{N}}$ and $D_{\sigma}=\alpha D_{t}+D_{x_{N}}$; the direction determined by $D_{\tau}$ is called the direction conjugate to the hyperplane $\left\{t=\alpha x_{N}\right\}$. Then

$$
D_{\tau}^{2}-D_{\sigma}^{2}=\left(1-\alpha^{2}\right)\left(D_{t}^{2}-D_{x_{N}}^{2}\right) .
$$

Remark 5.1 applies here too, showing that

$$
W \text { is constant along any line parallel to } l \text {, i.e., } D_{\sigma} W=0 \text {. }
$$

Hence, from (5.12) we get, setting $D_{s}=\left(1-\alpha^{2}\right)^{-1 / 2} D_{\tau}$ and $W^{\prime}\left(x_{1}, \ldots, x_{N-1}, s\right)=$ $W(x, t)$,

$$
D_{s}^{2} W^{\prime}-\Delta^{\prime} W^{\prime}=A\left(W^{\prime}\right)^{p},
$$

where $\Delta^{\prime}=\sum_{i=1}^{N-1} \partial^{2} / \partial x_{i}^{2}$; notice that

$$
\sigma=\frac{x_{N}-\alpha t}{1-\alpha^{2}}, \quad \tau=\frac{-\alpha x_{N}+t}{1-\alpha^{2}}, \quad s=\frac{-\alpha x_{N}+t}{\left(1-\alpha^{2}\right)^{1 / 2}} .
$$

$W^{\prime}$ also satisfies (4.12)-(4.15), (4.16) with the same $\varepsilon_{0}$, and is a convex function.

Applying the inductive assumption to $W^{\prime}$ we deduce that

$$
\begin{aligned}
& \tilde{\Gamma}_{0} \text { is an }(N-1) \text {-plane, and } \\
& W^{\prime}=\hat{C}_{\alpha} d^{-q}\left(\cdot, \tilde{\Gamma}_{0}\right), \quad \hat{C}_{\alpha}=C_{\alpha}\left(1+\alpha^{2}\right)^{-q / 2} .
\end{aligned}
$$

We can now show that

$$
\begin{aligned}
& \text { at any point of a generatrix } l \text { to } \hat{\Gamma} \text { there is a unique tangent } \\
& \text { plane } T_{l} \text { to } \hat{\Gamma} \text {. }
\end{aligned}
$$

Indeed, suppose $\Pi_{1}$ and $\Pi_{2}$ are two tangent hyperplanes. Then each $\Pi_{i}$ is still tangent to $\tilde{\Gamma}$ and thus, by (6.4), (6.6), it must coincide with the hyperplane $l_{0} \times \tilde{\Gamma}_{0}$.

From (6.7) we have that

$$
\tilde{\Gamma} \text { is differentiable at each of its points. }
$$

Take any generatrix $l$ and let $T_{l}$ be the hyperplane which supports $\hat{\Omega}$ at 0 and contains $l$. We now fix a coordinate system such that

$$
T_{l}:\left\{t=\alpha x_{N},\left(x_{1}, \ldots, x_{N-1}, x_{N}\right) \in \mathbf{R}^{N}\right\} .
$$

Set

$$
V_{l}(x, t)=C_{\alpha}\left(\alpha x_{N}-t\right)^{-q} .
$$

Let $l^{\prime}$ be any straight line parallel to $l$. In view of (6.6), $w-V_{l} \rightarrow 0$ if $(x, t) \rightarrow \infty$ along $l^{\prime}$ (with $t \rightarrow-\infty$ ). Also $D_{l l}\left(w-V_{l}\right)=D_{l l^{w}} \geqslant 0$ along $l^{\prime}$. It follows that

$$
w \geqslant V_{l} \text { in } \hat{\Omega} \text {. }
$$


We shall now establish an extension of Lemma 5.4. Choose again the $x_{i}$ coordinates such that (6.9) holds. Introducing $D_{\tau}$ and $D_{\sigma}$ as before we have

$$
\begin{aligned}
& \square w=\left(1-\alpha^{2}\right) D_{\tau}^{2} w-\left(1-\alpha^{2}\right) D_{\sigma}^{2}-\Delta^{\prime} w=A w^{p}, \\
& \square V_{l}=\left(1-\alpha^{2}\right) D_{\tau}^{2} V_{l}=A V_{l}^{p} .
\end{aligned}
$$

Let $m$ be any ray with initial point on $l$, in the direction conjugate normal to $T_{l}$, i.e., the direction $\tilde{v}=\alpha e_{N}+e_{N+1}$, where $e_{i}$ is the unit vector in the positive $x_{i}$-axis $\left(x_{N+1}=t\right)$. For any $\varepsilon>0$ let $V_{l}^{\varepsilon}$ and $l^{\varepsilon}$ be translations of $V_{l}$ and $l$ downward by $\varepsilon$. Then $V_{l}^{\varepsilon}=\infty>w$ at $m \cap l^{\varepsilon}, V_{l}^{\varepsilon}-w \rightarrow 0$ along $m$ as $(x, t) \rightarrow \infty$ and, by (6.12),

$$
\left(1-\alpha^{2}\right) D_{\tau}^{2}\left(w-V_{l}^{\varepsilon}\right) \geqslant A\left(w^{p}-\left(V_{l}^{\varepsilon}\right)^{p}\right) \text {. }
$$

Applying the maximum principle along $m$, we get $w \leqslant V_{l}^{\varepsilon}$ and, as $\varepsilon \rightarrow 0$,

$$
w \leqslant V_{l} \text { along } m \text {. }
$$

Recalling (6.11) we conclude that

$$
\begin{aligned}
& w=V_{l} \text { in the 2-plane sector (in } \hat{\Omega} \text { ) } \Pi_{l} \text { generated by } l \text { and the } \\
& \text { conjugate normal to } T_{l} \text {. }
\end{aligned}
$$

REMARK 6.1. The conjugate normal to $T_{l}$ is time-like (in the original coordinates). Indeed, this follows from the fact that $\tilde{\nu}=\alpha e_{N}+e_{N+1}(|\alpha|<1)$ in the special coordinates above, whereas the two coordinate systems are related by a rotation in the $x$-space only.

We now represent the cone $\hat{\Gamma}$ in polar coordinates $(\theta, \rho)$ by $\hat{\Gamma}: t=h(\theta) \rho, \rho<0$. Assume that $h_{0}=\min _{\theta} h(\theta)$ is taken at $\theta=e_{N}$. Then

$$
T \equiv\left\{t=h_{0} x_{N},\left(x_{1}, \ldots, x_{N-1}, x_{N}\right) \in \mathbf{R}^{N}\right\}
$$

is the support hyperplane to the cone $\hat{\Gamma}$ along the generatrix $\mu$ projecting on $e_{N}$, since both $T$ and $\hat{\Gamma}$ have zero differential in $\theta$ ( $\hat{\Gamma}$ is differentiable, by (6.8)) and they coincide along $\mu$. By (6.14) it then follows that $w=V_{\mu}$ in the 2-plane sector (in $\hat{\Omega}$ ) $\Pi_{\mu}$ generated by $(\mu, \sigma)$, where $\sigma$ is the conjugate normal to $T$, that is, $\sigma=h_{0} e_{N}+$ $e_{N+1}$. Since both $\mu$ and $\sigma$ are in the $\left(e_{N}, e_{N+1}\right)$-plane, $\Pi_{\mu}$ is a vertical sector; thus

$$
\begin{aligned}
w=V_{\mu} & \text { in } \Pi_{\mu}, \\
& \Pi_{\mu}=\left\{\left(0, \ldots, 0, x_{N}, t\right), x_{N} / \alpha<t<\alpha x_{N}, x_{N}>0\right\},
\end{aligned}
$$

where we have taken $\alpha=h_{0}>0$.

We now proceed analogously to Lemma 5.5. Take any two cones in $\hat{\Omega}$,

$$
K_{\bar{x}, \bar{i}, \delta}=\{(x, t) ;|x-\bar{x}|<\bar{t}-t, \bar{t}-\delta<t<\bar{t}\}
$$

and

$$
\hat{K}_{\bar{x}, \bar{t}, \delta}=\{(x, t) ;|x-\bar{x}|<t-\overline{\bar{t}}, \overline{\bar{t}}<t<\overline{\bar{t}}+\delta\},
$$

where $\bar{x}=\left(0, \ldots, 0, \bar{x}_{N}\right), \overline{\bar{t}}=\bar{t}-2 \delta, \delta>0$, and $(\bar{x}, \bar{t}),(\bar{x}, \overline{\bar{t}}) \in \Pi_{\mu}$. Take for definiteness $N=3$, and let $t_{0}=\bar{t}-\delta$. Represent $w\left(x, t_{0}+\lambda\right)$ and $w\left(x, t_{0}-\lambda\right)$ by $\left(1.2_{3}\right)$, using the Cauchy data at $t=t_{0}$, and add the corresponding expressions. 
Integrating with respect to $\lambda, 0<\lambda<\delta$, we obtain

$$
\begin{aligned}
\int_{-\delta}^{\delta} w\left(\bar{x}, t_{0}\right. & +\lambda) d \lambda=\frac{\delta}{2 \pi} \int_{|\xi|=1} w\left(\bar{x}+\delta \xi, t_{0}\right) d \omega_{\xi}+\frac{1}{4 \pi} \int_{0}^{\delta} d \lambda \int_{0}^{\lambda}(\lambda-s) d s \\
& \times \int_{|\eta|=1} A\left[w^{p}\left(\bar{x}+(\lambda-s) \eta, t_{0}+s\right)+w^{p}\left(\bar{x}+(\lambda-s) \eta, t_{0}-s\right)\right] d \omega_{\eta}
\end{aligned}
$$

Similar representation holds for $V_{\mu}$. By $(6.15) w(\bar{x}, \tau)=V_{\mu}(\bar{x}, \tau)$ if $\overline{\bar{t}} \leqslant \tau \leqslant \bar{t}$. Since, further, $w \geqslant V_{\mu}$ in $\hat{\Omega}$ (by (6.11)), we conclude from (6.16) that

$$
w=V_{\mu} \text { in } K_{\bar{x}, \bar{t}, \delta} \cup \hat{K}_{\bar{x}, \bar{t}, \delta} .
$$

By varying $\bar{x}, \bar{t}, \delta$ we can establish that $w=V_{\mu}$ in a cone $K^{*} \equiv\{|x|<t-c$, $-\infty<t<-\sigma\}$ for some positive constants $c, \sigma$.

Since any generatrix $l$ of $\hat{\Gamma}$ is space-like and its conjugate time-like (see Remark 6.1) the sector $\Pi_{l}$ defined in (6.14) must intersect the cone $K^{*}$. But then, by comparing (6.17) with (6.14) we deduce that $V_{l} \equiv V_{\mu}$ for any $l$, which means that $\hat{\Gamma}$ is planar. Thus (6.2) is valid and then also (6.1) holds.

7. Continuous differentiability of the blow-up boundary. In this section we complete the proof of Theorem 1.1. Let $\left(x_{0}, t_{0}\right)$ be any point of $\Gamma$ and take any blow-up limit

$$
u_{\lambda_{n}} \rightarrow v
$$

By Lemma 6.1, $v$ is a plane solution, i.e.,

$$
v=V_{\alpha}
$$

and $\{v<\infty\}$ is a half-space $\Omega_{0}$. Denote the tangent plane by $\Gamma_{0}$ and its inner normal by $N_{0}$.

For any $\varepsilon>0$ denote by $S_{\varepsilon}$ the set of all unit directions $\tau$ with $\tau \cdot N_{0} \geqslant \varepsilon$. For any $\tau \in S_{\varepsilon}$ consider the functions $U_{n}(x, t)=\partial u_{\lambda_{n}}(x, t) / \partial \tau$ in the domain

$$
G_{\delta}=B_{1} \cap \Omega_{0} \cap\left\{\operatorname{dist}\left((x, t), \Gamma_{0}\right)>\delta\right\},
$$

where $\delta$ is any small positive constant.

Since the convergence in $(7.1)$ is in the $C^{3, \beta}\left(G_{\delta}\right)$ sense (for any $0<\beta<1$ ), we certainly have that

$$
\begin{array}{ll}
U_{n} \rightarrow V_{\alpha, \tau}, & \nabla_{x} U_{n} \rightarrow \nabla_{x} V_{\alpha, \tau}, \\
D_{t} U_{n} \rightarrow D_{t} V_{\alpha, \tau} & \text { uniformly in } G_{\delta} .
\end{array}
$$

Now, the condition $\left(1.8_{3}\right)$ holds for $V_{\alpha, \tau}$ if the initial conditions are taken in the base of a cone $K^{*}$ for which the base lies in $G_{\delta}$; the same is then true of $U_{n}$ if $n \geqslant n_{0}$. But for some small $\rho>0$, every point $(x, t) \in B_{\rho}$ with $u_{\lambda_{n}}(x, t)<\infty$ can be taken as a vertex of such a cone $K^{*}$ with $u_{\lambda_{n}}<\infty$ in $K^{*}$. Appealing to the representation (1.2) we deduce that $U_{n}(x, t)>0$ if $n \geqslant n_{0}$. Thus, in particular,

$$
\frac{\partial}{\partial \tau} u_{\lambda_{n_{0}}}>0 \text { in } B_{\rho} \cap\left\{u_{\lambda_{n_{0}}}<\infty\right\}
$$


which means that

$$
\frac{\partial u}{\partial \tau}>0 \text { in } B_{\lambda_{\lambda_{0}^{\rho}}}\left(x_{0}, t_{0}\right) \cap \Omega \forall \tau \in S_{\varepsilon} .
$$

It follows that the blow-up surface in $B_{\lambda_{n \rho} \rho}\left(x_{0}, t_{0}\right)$ is a graph in each direction $\tau$ of $S_{\varepsilon}$. Consequently, the Lipschitz surface $t^{0}=\phi(x)$ satisfies $\left|D_{i} \phi(x)-D_{i} \phi\left(x^{\prime}\right)\right| \leqslant$ $C \varepsilon$ for a.e. points $x, x^{\prime}$ in some ball $B_{\sigma}\left(x_{0}\right)$, with $\sigma>0$. This implies that $\phi$ is continuously differentiable.

REMARK 7.1. From the above proof it follows that for the full blow-up family $u_{\lambda}$ there holds $u_{\lambda} \rightarrow V_{\alpha}$ in $C^{3, \beta}$ in compact subsets of $\left\{V_{\alpha}<\infty\right\}$. This implies that

$$
\frac{u_{t}^{2}-\left|\nabla_{x} u\right|^{2}}{u^{p+1}} \rightarrow \frac{2 A}{p+1} \quad \text { as } d(x, t) \rightarrow 0,(x, t) \rightarrow\left(x_{0}, \phi\left(x_{0}\right)\right) \text {, }
$$

and

$$
\begin{aligned}
& \frac{u_{t t}}{u^{p}} \rightarrow \frac{A}{1-\alpha^{2}}, \quad \frac{u_{t}^{2}}{u^{p+1}} \rightarrow \frac{2 A}{p+1} \frac{1}{1-\alpha^{2}}, \quad \alpha=\left|\nabla \phi\left(x_{0}\right)\right|, \\
& u d^{q} \rightarrow A_{0}\left(\frac{1-\alpha^{2}}{1+\alpha^{2}}\right)^{1 /(p-1)}, \quad A_{0}^{p-1}=\frac{1}{A} \frac{2(p+1)}{(p-1)^{2}} .
\end{aligned}
$$

REMARK 7.2. The proof of Theorem 1.1 extends to the case where $F=F(x, u)$.

REMARK 7.3. For $N=1$ Theorem 1.1 can be established assuming on $f, g$ only the condition (1.7); some monotonicity properties of $\phi(x)$ can also be established. This will appear in another publication (Differentiability of the blow-up curve for one dimensional nonlinear wave equations, in Arch. Rational Mech. Anal.).

\section{REFERENCES}

1. R. Glassey, Blow-up theorems for nonlinear wave equations, Math. Z. 132 (1973), 183-203.

2. __ Finite-time blow-up for solutions of nonlinear wave equations, Math. Z. 177 (1981), 323-340.

3. F. John, Blow-up of solutions of nonlinear wave equations in three space dimensions, Manuscripta Math. 28 (1979), 235-268.

4. H. Levine, Instability and nonexistence of global solutions to nonlinear wave equations of the form $P u_{t t}=-A u+F(u)$, Trans. Amęr. Math. Soc. 192 (1974), 1-21.

5. T. Kato, Blow-up of solutions of some nonlinear hyperbolic equations, Comm. Pure Appl. Math. 32 (1980), 501-505.

Department of Mathematics, University of Chicago, Chicago, Illinois 60637

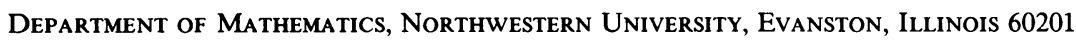

